PROCEEDINGS OF THE

AMERICAN MATHEMATICAL SOCIETY

Volume 135, Number 11, November 2007, Pages 3621-3630

S 0002-9939(07)08893-4

Article electronically published on August 1, 2007

\title{
SOME EXACT SEQUENCES FOR TOEPLITZ ALGEBRAS OF SPHERICAL ISOMETRIES
}

\author{
BEBE PRUNARU
}

(Communicated by Joseph A. Ball)

\begin{abstract}
A family $\left\{T_{j}\right\}_{j \in J}$ of commuting bounded operators on a Hilbert space $H$ is said to be a spherical isometry if $\sum_{j \in J} T_{j}^{*} T_{j}=1$ in the weak operator topology. We show that every commuting family $\mathcal{F}$ of spherical isometries is jointly subnormal, which means that it has a commuting normal extension $\widehat{\mathcal{F}}$ on some Hilbert space $\widehat{H} \supset H$. Suppose now that the normal extension $\widehat{\mathcal{F}}$ is minimal. Then we show that every bounded operator $X$ in the commutant of $\mathcal{F}$ has a unique norm preserving extension to an operator $\widehat{X}$ in the commutant of $\widehat{\mathcal{F}}$. Moreover, if $\mathcal{C}$ is the commutator ideal in $C^{*}(\mathcal{F})$, then $C^{*}(\mathcal{F}) / \mathcal{C}$ is ${ }^{*}$-isomorphic to $C^{*}(\widehat{\mathcal{F}})$. We also show that the commutant of the minimal normal extension is completely isometric, via the compression mapping, to the space of Toeplitz-type operators associated to $\mathcal{F}$. We apply these results to construct exact sequences for Toeplitz algebras on generalized Hardy spaces associated to strictly pseudoconvex domains.
\end{abstract}

\section{INTRODUCTION}

Consider a complex Hilbert space $\mathcal{H}$ and let $\mathcal{B}(\mathcal{H})$ denote the algebra of all bounded linear operators on $\mathcal{H}$. A commutative family $\mathcal{S}=\left\{T_{j}\right\}_{j \in J}$ of bounded operators on $\mathcal{H}$ is said to be a spherical isometry if $\sum_{j \in J} T_{j}^{*} T_{j}=1$ in the weak operator topology. For instance, the multiplication operators $\left\{T_{z_{1}}, \ldots, T_{z_{n}}\right\}$ on the Hardy space $H^{2}\left(S^{2 n-1}\right)$ of the unit sphere in $\mathbb{C}^{n}$ form a spherical isometry which is called the Szegö $n$-tuple on $S^{2 n-1}$.

Spherical isometries, mostly with a finite number of components, have been recently studied in a number of papers; see [At90, At98, AL96, Did05, Es99, [Es01, Es06], EsP01]. In At90 it was proved that every spherical isometry with a finite number of terms is subnormal in the sense that it has a commuting normal extension. The main purpose of the present paper is to extend this result to an arbitrary family of commuting spherical isometries with a finite or even an infinite number of components. Our approach is completely different from that in At90, and it is mainly based on the study of the space of Toeplitz-type operators associated to spherical isometries. We show that this space is the range of a completely

Received by the editors April 3, 2006 and, in revised form, August 22, 2006.

2000 Mathematics Subject Classification. Primary 47L80, 47B35; Secondary 47B20, 46L07.

Key words and phrases. Toeplitz operators, Toeplitz algebras, spectral inclusion, spherical isometry, completely positive projection, injective operator spaces, strictly pseudoconvex domains.

This research was partially supported by the Romanian Ministry of Education and Research, through the grant CEx05-D11-23/2005. 
positive idempotent mapping on $\mathcal{B}(\mathcal{H})$ and use the properties of this mapping to construct simultaneous normal extensions via the Stinesprig Dilation Theorem. On the way, we also obtain some exact sequences that relate the $C^{*}$-algebras generated by spherical isometries to those generated by their minimal normal extensions.

Our main object of study in what follows is a commuting family of spherical isometries $\mathcal{F}=\left\{\mathcal{S}_{\alpha}\right\}_{\alpha \in \Gamma}$ on some Hilbert space $\mathcal{H}$. This means that if $\alpha \in \Gamma$, then $\mathcal{S}_{\alpha}=\left\{T_{j, \alpha}\right\}_{j \in J_{\alpha}}$ is a spherical isometry and the union $\bigcup_{\alpha \in \Gamma} \mathcal{S}_{\alpha}$ is a commutative set of operators. To each such family we associate its space of Toeplitz operators as follows.

Definition 1.1. Given a commuting family $\mathcal{F}=\left\{\mathcal{S}_{\alpha}\right\}_{\alpha \in \Gamma}$ of spherical isometries on $\mathcal{H}$ we define, using the notations above, the space $\mathcal{T}(\mathcal{F})$ of all $\mathcal{F}$-Toeplitz operators to be the set of all operators $X \in \mathcal{B}(\mathcal{H})$ such that

for all $\alpha \in \Gamma$.

$$
\sum_{j \in J_{\alpha}} T_{j, \alpha}^{*} X T_{j, \alpha}=X
$$

This terminology is inspired from the classical case of the unilateral shift $S$ on the Hardy space $H^{2}$ of the unit circle, where by a well-known result of A. Brown and P. Halmos (see BH63]) the solutions of the operator equation $S^{*} X S=X$ are precisely the Toeplitz operators on $H^{2}$. The main result of this paper is the following:

Theorem 1.2. Let $\mathcal{F}=\left\{\mathcal{S}_{\alpha}\right\}_{\alpha \in \Gamma}$ be a commuting family of spherical isometries on some Hilbert space $\mathcal{H}$ with $\mathcal{S}_{\alpha}=\left\{T_{j, \alpha}\right\}_{j \in J_{\alpha}}$ for each $\alpha \in \Gamma$, and let $\mathcal{T}(\mathcal{F})$ be the space of all $\mathcal{F}$-Toeplitz operators (see Definition 1.1 above). Also let $C^{*}(\mathcal{T}(\mathcal{F})$ ) denote the $C^{*}$-subalgebra of $\mathcal{B}(\mathcal{H})$ generated by $\mathcal{T}(\mathcal{F})$. Then we have:

(1) There exists a completely positive unital mapping $\Phi: \mathcal{B}(\mathcal{H}) \rightarrow \mathcal{B}(\mathcal{H})$ such that $\Phi \circ \Phi=\Phi$ and $\operatorname{Ran} \Phi=\mathcal{T}(\mathcal{F})$. Moreover, for every $X \in \mathcal{B}(\mathcal{H})$ and every operator $A, B$ in the commutant of $\mathcal{F}$ we have $\Phi\left(A^{*} X B\right)=$ $A^{*} \Phi(X) B$.

(2) There exist a Hilbert space $\widehat{\mathcal{H}} \supset \mathcal{H}$ and a commuting family $\tilde{\mathcal{F}}=\left\{\widehat{\mathcal{S}}_{\alpha}\right\}_{\alpha \in \Gamma}$ of normal spherical isometries on $\widehat{\mathcal{H}}$ with $\widehat{\mathcal{S}}_{\alpha}=\left\{\widehat{T}_{j, \alpha}\right\}_{j \in J_{\alpha}}$ which leaves $\mathcal{H}$ invariant and whose restriction to $\mathcal{H}$ coincides with $\mathcal{F}$.

(3) Suppose now that the normal extension $\tilde{\mathcal{F}}$ is minimal, i.e. $\widehat{\mathcal{H}}$ is the smallest reducing subspace for $\tilde{\mathcal{F}}$ containing $\mathcal{H}$. Then there exists a unital *representation $\pi: C^{*}(\mathcal{T}(\mathcal{F})) \rightarrow \mathcal{B}(\widehat{\mathcal{H}})$ with the following properties:

(3a) $\pi\left(T_{j, \alpha}\right)=\widehat{T}_{j, \alpha}$ for all $\alpha \in \Gamma$ and $j \in J_{\alpha}$.

(3b) If $P_{\mathcal{H}}$ is the orthogonal projection of $\widehat{\mathcal{H}}$ onto $\mathcal{H}$, then

$$
\Phi(X)=\left.P_{\mathcal{H}} \pi(X)\right|_{\mathcal{H}}
$$

for every $X \in C^{*}(\mathcal{T}(\mathcal{F}))$.

(3c) Its image $\pi\left(C^{*}(\mathcal{T}(\mathcal{F}))\right)$ coincides with the commutant in $\mathcal{B}(\widehat{\mathcal{H}})$ of the unital $C^{*}$-algebra $C^{*}(\tilde{\mathcal{F}})$ generated by $\tilde{\mathcal{F}}$ in $\mathcal{B}(\widehat{\mathcal{H}})$, so it is a type I von Neumann algebra.

(3d) Its kernel coincides with the two-sided closed ideal of $C^{*}(\mathcal{T}(\mathcal{F}))$ generated by all operators of the form $X Y-\Phi(X Y)$ with $X, Y \in \mathcal{T}(\mathcal{F})$.

(3e) The mapping $\rho: \pi\left(C^{*}(\mathcal{T}(\mathcal{F}))\right) \rightarrow \mathcal{B}(\mathcal{H})$ defined by

$$
\rho(\pi(X))=\left.P_{\mathcal{H}} \pi(X)\right|_{\mathcal{H}}
$$


is a complete isometry onto the space $\mathcal{T}(\mathcal{F})$ such that $\pi \circ \rho$ is the identity on $\pi\left(C^{*}(\mathcal{T}(\mathcal{F}))\right)$.

(3f) Let $C^{*}(\mathcal{F})$ denote the unital $C^{*}$-algebra generated by $\mathcal{F}$ in $\mathcal{B}(\mathcal{H})$, and let $\mathcal{C}$ be the closed ideal of $C^{*}(\mathcal{F})$ generated by all the commutators $X Y-Y X$ with $X, Y \in \mathcal{T}(\mathcal{F}) \cap C^{*}(\mathcal{F})$. Let $\pi_{0}$ be the restriction of $\pi$ to $C^{*}(\mathcal{F})$ and let $\rho_{0}$ denote the restriction of $\rho$ to $C^{*}(\tilde{\mathcal{F}})$. Then there exists a short exact sequence

$$
0 \rightarrow \mathcal{C} \hookrightarrow C^{*}(\mathcal{F}) \stackrel{\pi_{0}}{\longrightarrow} C^{*}(\tilde{\mathcal{F}}) \rightarrow 0
$$

for which $\rho_{0}$ is a completely isometric cross section.

$(3 \mathrm{~g})$ An operator $X \in \mathcal{B}(\mathcal{H})$ belongs to the commutant of $\mathcal{F}$ if and only if both $X$ and $X^{*} X$ belong to $\mathcal{T}(\mathcal{F})$. In this case there exists a unique operator $\widehat{X}$ in the commutant of $\tilde{\mathcal{F}}$ which leaves $\mathcal{H}$ invariant and whose restriction to $\mathcal{H}$ coincides with $X$. Moreover the map $X \mapsto \widehat{X}$ is norm preserving.

This paper is organized as follows. The next section contains some preliminary results concerning completely positive projections. The proof of Theorem 1.2 will be given in the third section. In the last section we use Theorem 1.2 in order to construct exact sequences for Toeplitz algebras on strictly pseudoconvex domains.

\section{Some PRELIMINARY RESUlts}

We begin by recalling for later use the following well-known result from [CE77.

Theorem 2.1. Let $\Phi: \mathcal{B}(\mathcal{H}) \rightarrow \mathcal{B}(\mathcal{H})$ be a completely positive and completely contractive mapping such that $\Phi \circ \Phi=\Phi$. Then for all $X, Y \in \mathcal{B}(\mathcal{H})$

$$
\Phi(\Phi(X) Y)=\Phi(X \Phi(Y))=\Phi(\Phi(X) \Phi(Y)) \text {. }
$$

In CE77] this result is used to show that when $\Phi$ is also unital, its range $\operatorname{Ran} \Phi$ can be endowed with a $C^{*}$-algebra structure for which the identity mapping on $\operatorname{Ran} \Phi$ is completely isometric. The multiplication is defined by the rule

$$
\Phi(X) \circ \Phi(Y)=\Phi(\Phi(X) \Phi(Y)) .
$$

For our purposes we need a concrete realization of this $C^{*}$-algebra as an operator algebra on some Hilbert space. This is quite obviously provided through the use of the Stinespring Dilation Theorem [St55] as follows.

Lemma 2.2. Let $\Phi: \mathcal{B}(\mathcal{H}) \rightarrow \mathcal{B}(\mathcal{H})$ be a completely positive, unital mapping such that $\Phi \circ \Phi=\Phi$, let $\mathcal{E}=\operatorname{Ran} \Phi$ and let $\Phi_{0}: C^{*}(\mathcal{E}) \rightarrow \mathcal{B}(\mathcal{H})$ be its restriction to the $C^{*}$-algebra generated by $\mathcal{E}$ in $\mathcal{B}(\mathcal{H})$. Suppose that $\pi: C^{*}(\mathcal{E}) \rightarrow \mathcal{B}(\mathcal{K})$ is its minimal Stinespring dilation so that $\Phi_{0}(X)=V^{*} \pi(X) V$ for some isometry $V: \mathcal{H} \rightarrow \mathcal{K}$ and for all $X \in C^{*}(\mathcal{E})$. Then $\operatorname{Ker} \Phi_{0}=\operatorname{Ker} \pi$ and the mapping $\rho: \pi\left(C^{*}(\mathcal{E})\right) \rightarrow \mathcal{B}(\mathcal{H})$ defined by $\rho(\pi(X))=V^{*} \pi(X) V$ for $X \in C^{*}(\mathcal{E})$ is a complete isometry such that $\pi \circ \rho$ is the identity on $\pi\left(C^{*}(\mathcal{E})\right)$ and $\operatorname{Ran} \rho=\operatorname{Ran} \Phi$. Moreover, if $\operatorname{Ran} \Phi$ is $\sigma$-weakly closed, then $\pi\left(C^{*}(\mathcal{E})\right)$ is also $\sigma$-weakly closed, hence a von Neumann subalgebra of $\mathcal{B}(\mathcal{K})$, and the map $\rho$ defined above is a $\sigma$-weak homeomorphism.

Proof. To begin with, one can see from Theorem 2.1 above that $\operatorname{Ker} \Phi_{0}$ is an ideal in $C^{*}(\mathcal{E})$. Now, it is a general fact, and easy to prove, that if the kernel of a completely positive map defined on a $C^{*}$-algebra is an ideal, then it equals the kernel of its minimal Stinespring dilation. We now show that $\rho$ is a complete isometry. First, $\rho$ is one-to-one because $\operatorname{Ker} \pi=\operatorname{Ker} \Phi_{0}$. Moreover, since $\Phi_{0} \circ \Phi_{0}=\Phi_{0}$, we have that 
$\rho \circ \pi \circ \rho \circ \pi=\rho \circ \pi$ hence $\pi \circ \rho$ is the identity on $\pi\left(C^{*}(\mathcal{E})\right)$. It then follows, since both $\pi$ and $\rho$ are completely contractive, that $\rho$ is actually completely isometric.

Suppose now that $\mathcal{E}$ is $\sigma$-weakly closed. In this case, it turns out that $\pi\left(C^{*}(\mathcal{E})\right)$ is isometric with a dual Banach space, namely $\mathcal{E}$, therefore it is a $W^{*}$-algebra whose weak $^{*}$ topology is the one induced from $\mathcal{E}$ via the mapping $X \mapsto \pi(X)$. Therefore a net $\left\{Y_{\lambda}\right\}$ in $\pi\left(C^{*}(\mathcal{E})\right)$ converges to zero in this topology if and only if $\rho\left(Y_{\lambda}\right) \rightarrow 0$ weak $^{*}$ in $\mathcal{B}(\mathcal{H})$. Moreover, since the multiplication in a $W^{*}$-algebra is separately weak* continuous, it follows in this case that for every $Z_{1}, Z_{2} \in C^{*}(\mathcal{E})$ we also have that $\rho\left(\pi\left(Z_{1}\right) Y_{\lambda} \pi\left(Z_{2}\right)\right) \rightarrow 0$ weak $^{*}$ in $\mathcal{B}(\mathcal{H})$. In order to prove that $\pi\left(C^{*}(\mathcal{E})\right)$ is $\sigma$ weakly closed it suffices to show that the mapping $X \mapsto \pi(X)$ (which is an isometry on $\mathcal{E}$ ) is continuous when both $\mathcal{E}$ and $\mathcal{B}(\mathcal{K})$ are endowed with their corresponding weak* topologies. Moreover, it is enough to check this continuity only on bounded subsets of $\mathcal{E}$. Let $\left\{X_{\lambda}\right\}$ be a bounded net weak* convergent to 0 . By what we have already shown above, it follows that for each $Z_{1}, Z_{2} \in C^{*}(\mathcal{E})$ and $h_{1}, h_{2} \in \mathcal{H}$ we have that

$$
\left(\pi\left(X_{\lambda}\right) \pi\left(Z_{1}\right) V h_{1}, \pi\left(Z_{2}\right) V h_{2}\right) \rightarrow 0
$$

which shows that $\pi\left(X_{\lambda}\right) \rightarrow 0$ weak $^{*}$ in $\mathcal{B}(\mathcal{K})$ because $\pi\left(X_{\lambda}\right)$ is bounded and $\pi$ is minimal. This completes the proof.

The following lemma is a particular case of a more general result proved in BP05.

Lemma 2.3. Let $\left\{\phi_{\alpha}\right\}_{\alpha \in \Gamma}$ be a set of commuting completely positive unital and normal mappings acting on $\mathcal{B}(\mathcal{H})$ for some Hilbert space $\mathcal{H}$. Then there exists a completely positive mapping $\Phi: \mathcal{B}(\mathcal{H}) \rightarrow \mathcal{B}(\mathcal{H})$ whose range is precisely the set

$$
\left\{X \in \mathcal{B}(\mathcal{H}): \phi_{\alpha}(X)=X, \alpha \in \Gamma\right\}
$$

and such that $\Phi \circ \Phi=\Phi$.

Sketch of the proof. Let $S$ denote the semigroup generated by the set $\left\{\phi_{\alpha}\right\}_{\alpha \in \Gamma}$. Each element $s \in S$ correspond to a completely positive unital and normal mapping $\psi_{s}: \mathcal{B}(\mathcal{H}) \rightarrow \mathcal{B}(\mathcal{H})$ which is a finite product of $\phi_{\alpha}$. It is obvious that the fixed point set of $\left\{\phi_{\alpha}\right\}_{\alpha \in \Gamma}$ is the same as that of $\left\{\psi_{s}\right\}_{s \in S}$. We thus obtain an action

$$
\gamma: S \times \mathcal{B}(\mathcal{H}) \rightarrow \mathcal{B}(\mathcal{H})
$$

defined by $\gamma(s, X)=\psi_{s}(X)$ for all $s \in S$ and $X \in \mathcal{B}(\mathcal{H})$. Since $S$ is commutative, a well-known result of Dixmier Dix50] shows that $S$ is amenable, which means that there exists a state $\mu$ on the $C^{*}$-algebra $\ell^{\infty}(S)$ of all bounded complex functions on $S$ which is invariant under all translations with elements from $S$. Given $T \in \mathcal{B}(\mathcal{H})$, for each pair of vectors $\xi, \eta \in \mathcal{H}$ define $[\xi, \eta]_{T}=\mu(\gamma(\cdot, T) \xi, \eta)$ and observe that this is a bounded sesquilinear map; therefore, there exists an operator $\Phi(T) \in \mathcal{B}(\mathcal{H})$ such that

$$
(\Phi(T) \xi, \eta)=[\xi, \eta]_{T}
$$

for all $\xi, \eta \in \mathcal{H}$. The rest of the proof is a matter of routine, and it will be left to the reader.

\section{Proof of the main Result}

Proof of Theorem 1.2. For each $\alpha \in \Gamma$ let $\phi_{\alpha}: \mathcal{B}(\mathcal{H}) \rightarrow \mathcal{B}(\mathcal{H})$ be defined by

$$
\phi_{\alpha}(X)=\sum_{j \in J_{\alpha}} T_{j, \alpha}^{*} X T_{j, \alpha}
$$


for all $X \in \mathcal{B}(\mathcal{H})$. It is not difficult to see that $\phi_{\alpha}$ is a completely positive unital and normal mapping. It follows that $\mathcal{T}(\mathcal{F})$ is precisely the set of common fixed points of the commuting family of mappings $\left\{\phi_{\alpha}\right\}_{\alpha \in \Gamma}$. Therefore we can apply Lemma 2.3 to infer the existence of an idempotent completely positive mapping $\Phi: \mathcal{B}(\mathcal{H}) \rightarrow \mathcal{B}(\mathcal{H})$ whose range is precisely $\mathcal{T}(\mathcal{F})$. Moreover, if $A, B$ are operators in the commutant of $\mathcal{F}$, then obviously $\phi_{\alpha}\left(A^{*} X B\right)=A^{*} \phi_{\alpha}(X) B$ for all $X \in \mathcal{B}(\mathcal{H})$ and all $\alpha \in \Gamma$. Therefore, as the proof of Lemma 2.3 shows, this holds true for $\Phi$ as well. This proves item (1).

Let $\Phi$ be as in item (1) and let $\Phi_{0}$ denote its restriction to $C^{*}(\mathcal{T}(\mathcal{F}))$. Denote by $\pi: C^{*}(\mathcal{T}(\mathcal{F})) \rightarrow \mathcal{B}(\widehat{\mathcal{H}})$ the minimal Stinespring dilation of $\Phi_{0}$. Therefore there exists an isometry $V: \mathcal{H} \rightarrow \widehat{\mathcal{H}}$ such that

$$
\Phi_{0}(X)=V^{*} \pi(X) V
$$

for all $X \in C^{*}(\mathcal{T}(\mathcal{F}))$. We see that we are precisely in the situation of Lemma 2.2 above, and moreover the range of $\Phi$ is also $\sigma$-weakly closed because it is the set of all common fixed points of a family of normal mappings. The conclusion that follows is that the mapping

$$
\rho: \pi\left(C^{*}(\mathcal{T}(\mathcal{F}))\right) \rightarrow \mathcal{B}(\mathcal{H})
$$

defined by $\rho(\pi(X))=V^{*} \pi(X) V$ for $X \in C^{*}(\mathcal{T}(\mathcal{F}))$ is a complete isometry onto the space of all $\mathcal{F}$-Toeplitz operators such that $\pi \circ \rho$ is the identity on $\pi\left(C^{*}(\mathcal{T}(\mathcal{F}))\right)$ and the latter is a von Neumann subalgebra of $\mathcal{B}(\widehat{\mathcal{H}})$. Let $\widehat{T}_{j, \alpha}=\pi\left(T_{j, \alpha}\right)$ for all $\alpha \in \Gamma$ and $j \in J_{\alpha}$, and also let $\widehat{\mathcal{S}}_{\alpha}=\left\{\widehat{T}_{j, \alpha}\right\}_{j \in J_{\alpha}}$ and let $\tilde{\mathcal{F}}=\left\{\widehat{\mathcal{S}}_{\alpha}\right\}_{\alpha \in \Gamma}$. Our next aim is to show that each family $\widehat{\mathcal{S}}_{\alpha}$ is a spherical isometry and that

$$
\sum_{j \in J_{\alpha}} \widehat{T}_{j, \alpha}^{*} \pi(X) \widehat{T}_{j, \alpha}=\pi(X)
$$

for all $X \in C^{*}(\mathcal{T}(\mathcal{F}))$. For this purpose, fix $\alpha \in \Gamma$ and observe that

$$
\sum_{j \in F} \widehat{T}_{j, \alpha}^{*} \widehat{T}_{j, \alpha} \leq 1
$$

for each finite subset $F \subset J_{\alpha}$. Therefore

$$
\sum_{j \in J_{\alpha}} \widehat{T}_{j, \alpha}^{*} \widehat{T}_{j, \alpha} \leq 1
$$

Now, let $X \in C^{*}(\mathcal{T}(\mathcal{F}))$, let

$$
Z=\sum_{j \in J_{\alpha}} \widehat{T}_{j, \alpha}^{*} \pi(X) \widehat{T}_{j, \alpha}
$$

and let $F \subset J_{\alpha}$ be a finite set. Then we see that, since $\pi(X)=\pi(\Phi(X))$ and since, by item (1), $\Phi\left(T_{j, \alpha}^{*} X T_{j, \alpha}\right)=T_{j, \alpha}^{*} \Phi(X) T_{j, \alpha}$, we have that

$$
\rho\left(\sum_{j \in F} \widehat{T}_{j, \alpha}^{*} \pi(X) \widehat{T}_{j, \alpha}\right)=\sum_{j \in F} T_{j, \alpha}^{*} \Phi(X) T_{j, \alpha}
$$

Taking weak*-limits in both sides, over the directed set of all finite subsets of $J_{\alpha}$, we get $\rho(Z)=\phi_{\alpha}(\Phi(X))=\Phi(X)=\rho(\pi(X))$. Since $\rho$ is one-to-one, we get $Z=\pi(X)$ which means that indeed

$$
\sum_{j \in J_{\alpha}} \widehat{T}_{j, \alpha}^{*} \pi(X) \widehat{T}_{j, \alpha}=\pi(X)
$$


for all $X \in C^{*}(\mathcal{T}(\mathcal{F}))$. Now, if $\pi(X)$ is an orthogonal projection, the previous identity implies that $\operatorname{Ker} \pi(X)$ is an invariant subspace for all $\widehat{T}_{j, \alpha}$. Since $\pi\left(C^{*}(\mathcal{T}(\mathcal{F}))\right)$ is a von Neumann algebra, this shows that all $\widehat{T}_{j, \alpha}$ belong to the center of $\pi\left(C^{*}(\mathcal{T}(\mathcal{F}))\right)$; in particular they are commuting normal operators. Since $T_{j, \alpha}=V^{*} \widehat{T}_{j, \alpha} V$ and both $\mathcal{S}_{\alpha}$ and $\widehat{\mathcal{S}}_{\alpha}$ are spherical isometries it is easy to see that $\widehat{T}_{j, \alpha} V \mathcal{H} \subset V \mathcal{H}$ for all $\alpha \in \Gamma$ and $j \in J_{\alpha}$. This shows that the family $\mathcal{F}$ is subnormal, which proves item (2).

We will show now that $\tilde{\mathcal{F}}$ is in fact the minimal normal extension of $\mathcal{F}$. For this purpose let $\mathcal{K}$ be the smallest reducing subspace for $\pi\left(C^{*}(\mathcal{F})\right)$ containing $V \mathcal{H}$ so we need to show that $\mathcal{K}=\widehat{\mathcal{H}}$. Let $\pi_{\mathcal{K}}: C^{*}(\mathcal{F}) \rightarrow \mathcal{B}(\mathcal{K})$ be the ${ }^{*}$-representation defined by $\pi_{\mathcal{K}}(X)=\left.P_{\mathcal{K}} \pi(X)\right|_{\mathcal{K}}$ where $P_{\mathcal{K}}$ denotes the orthogonal projection of $\widehat{\mathcal{H}}$ onto $\mathcal{K}$. We will show that the map defined by $\rho_{\mathcal{K}}(\pi(X))=\left.P_{\mathcal{K}} \pi(X)\right|_{\mathcal{K}}$ is a ${ }^{*}$-isomorphism of $\pi\left(C^{*}(\mathcal{T}(\mathcal{F}))\right)$ onto the commutant $\pi_{\mathcal{K}}\left(C^{*}(\mathcal{F})\right)^{\prime}$ of $\pi_{\mathcal{K}}\left(C^{*}(\mathcal{F})\right)$. In particular, this would imply, via the minimality of $\pi$, that $\mathcal{K}=\widehat{\mathcal{H}}$.

To begin with, it is clear that $\rho_{\mathcal{K}}$ is a completely positive and completely contractive mapping. It takes values in $\pi_{\mathcal{K}}\left(C^{*}(\mathcal{F})\right)^{\prime}$ because each $\widehat{T}_{j, \alpha}$ is in the center of $\pi\left(C^{*}(\mathcal{T}(\mathcal{F}))\right)$ and because the space $\mathcal{K}$ is reducing for all $\widehat{T}_{j, \alpha}$. Let

$$
\rho_{\mathcal{H}}: \pi_{\mathcal{K}}\left(C^{*}(\mathcal{F})\right)^{\prime} \rightarrow \mathcal{B}(\mathcal{H})
$$

be defined by $\rho_{\mathcal{H}}(Y)=V^{*} Y V$ for $Y \in \pi_{\mathcal{K}}\left(C^{*}(\mathcal{F})\right)^{\prime}$. Then it is obvious that $\operatorname{Ran} \rho_{\mathcal{H}} \subset \mathcal{T}(\mathcal{F})$ and moreover $\rho=\rho_{\mathcal{H}} \rho_{\mathcal{K}}$ where $\rho$ was defined above as $\rho(Y)=$ $V^{*} Y V$ for $Y \in \pi\left(C^{*}(\mathcal{T}(\mathcal{F}))\right)$. Recall now that we already proved that $\rho$ is completely isometric which implies that the mapping $\rho_{\mathcal{K}}$ is completely isometric. Therefore in order to show that $\rho_{\mathcal{K}}$ is onto, it suffices to observe that $\rho_{\mathcal{H}}$ is one-to-one on $\pi_{\mathcal{K}}\left(C^{*}(\mathcal{F})\right)^{\prime}$, and the latter follows easily from the minimality of $\pi_{\mathcal{K}}$. What we have now is that $\rho_{\mathcal{K}}$ is a unital complete isometry between two unital $C^{*}$-algebras. Since, by a well known result of Kadison Kad51, any such mapping is multiplicative, it follows that $\rho_{\mathcal{K}}$ is indeed a ${ }^{*}$-isomorphism of $\pi\left(C^{*}(\mathcal{T}(\mathcal{F}))\right)$ onto $\pi_{\mathcal{K}}\left(C^{*}(\mathcal{F})\right)^{\prime}$; in particular the space $\mathcal{K}$ is invariant under $\pi\left(C^{*}(\mathcal{T}(\mathcal{F}))\right)$. Since $\pi$ is minimal, this shows that $\mathcal{K}=\widehat{\mathcal{H}}$ hence $\tilde{\mathcal{F}}$ is the minimal normal extension of $\mathcal{F}$. So we have proved (3a), (3b), (3c) and (3e).

The proof of (3d) that $K e r \pi$ is the ideal generated by all operators of the form $X Y-\Phi(X Y)$ with $X, Y \in \mathcal{T}(\mathcal{F})$ follows by an easy induction argument on the length of an arbitrary product of elements from $\mathcal{T}(\mathcal{F})$ using the fact that

$$
\operatorname{Ker} \pi=\left\{X-\Phi(X): X \in C^{*}(\mathcal{T}(\mathcal{F}))\right\}
$$

together with Theorem 2.1 .

We now begin to prove (3f). For this purpose, we first show that $\Phi\left(C^{*}(\mathcal{F})\right)=$ $C^{*}(\mathcal{F}) \cap \mathcal{T}(\mathcal{F})$. Since $\Phi^{2}=\Phi$ it is enough to show that $\Phi\left(C^{*}(\mathcal{F})\right) \subset C^{*}(\mathcal{F})$. This inclusion follows easily from the fact that $\Phi$ takes any finite product of $T_{j, \alpha}$ and $T_{j, \alpha}^{*}$ into a permutation of the same product having all the $T_{j, \alpha}^{*}$ 's at the left and all the $T_{j, \alpha}$ 's at the right. Since $\Phi\left(C^{*}(\mathcal{F})\right) \subset C^{*}(\mathcal{F})$ we obtain that $\operatorname{Ker} \pi_{0}=$ $\left\{X-\Phi(X): X \in C^{*}(\mathcal{F})\right\}$. Now we can easily prove that the kernel of $\pi_{0}$ coincides with the closed ideal of $C^{*}(\mathcal{F})$ generated by all commutators $X Y-Y X$ with $X, Y \in C^{*}(\mathcal{F}) \cap \mathcal{T}(\mathcal{F})$. First, since $\pi_{0}\left(C^{*}(\mathcal{F})\right)$ is commutative, we have that any such commutator is in $\operatorname{Ker}\left(\pi_{0}\right)$. Now, if $X \in C^{*}(\mathcal{F})$ is a finite product of $T_{j, \alpha}$ and $T_{j, \alpha}^{*}$ it becomes obvious from the above description of $\Phi(X)$ that $X-\Phi(X)$ belongs 
to the ideal generated by all commutators $X Y-Y X$ with $X, Y \in C^{*}(\mathcal{F}) \cap \mathcal{T}(\mathcal{F})$. This completes the proof of $(3 \mathrm{f})$.

We now prove $(3 \mathrm{~g})$. If $X \in \mathcal{B}(\mathcal{H})$ is such that $X$ commutes with all operators from $\mathcal{F}$, then obviously $X$ and $X^{*} X$ belong to $\mathcal{T}(\mathcal{F})$. Suppose now that $X \in \mathcal{B}(\mathcal{H})$ is such that both $X$ and $X^{*} X$ belong to $\mathcal{T}(\mathcal{F})$. If $\widehat{X}=\pi(X)$, then $\widehat{X}$ commutes with all the normal extensions from $\tilde{\mathcal{F}}$ and $V^{*} \widehat{X} V=X$. Moreover $\|\widehat{X}\|=\|X\|$, so all we need to show is that $\widehat{X} V \mathcal{H} \subset V \mathcal{H}$. For this purpose, we observe that since $X^{*} X \in \mathcal{T}(\mathcal{F})$, then $X^{*} X=V^{*} \pi\left(X^{*} X\right) V=V^{*} \widehat{X}^{*} \widehat{X} V$. Therefore if $\xi \in \mathcal{H}$, then $\left\|V^{*} \widehat{X} V \xi\right\|=\|X \xi\|=\|\widehat{X} V \xi\|$ which implies that indeed $\widehat{X} V \mathcal{H} \subset V \mathcal{H}$. This finishes the proof of (3f) and the proof of the theorem as well.

Projections onto the space of Toeplitz operators on the Hardy space of the unit circle appear in several places; see for instance [Ar75]. Exact sequences for Toeplitz algebras associated to the unilateral shift $S$ on $H^{2}(\mathbb{T})$ have been constructed by L.A. Coburn and R.G. Douglas (see Chapter VII in Do98]). Similar results for the Szegö $n$-tuple on the unit sphere in $\mathbb{C}^{n}$ were proved in Co73] and DJ77. For the case of a commuting family of isometries, the existence of a commuting unitary extension was proved in Ito58 and the commutant lifting was proved by S. Brehmer and R.G. Douglas (see Do69]). Exact sequences for $C^{*}$-algebras generated by finite families of commuting isometries have been studied in [BCL78]. $C^{*}$-algebras generated by isometric representations of commuting semigroups have been studied mainly for semigroups of positive elements in ordered abelian groups; see [BC70], Do72, Mu87. For the case of a single finite spherical isometry the existence of a normal extension along with a commutant lifting theorem were proved in [At90; see also [AL96 for alternate proofs. A completely different proof of the subnormality of a spherical isometry appears in [Ar98.

\section{Applications to uniform algebras}

In this section we shall apply Theorem 1.2 to construct exact sequences for Toeplitz operators associated to uniform algebras on compact spaces. The following general framework is frequently used when dealing with Toeplitz operators on Hardy spaces; see for instance [TY78] or Mu92]. Let $K$ be a compact Hausdorff space and let $C(K)$ denote the Banach algebra of all complex-valued continuous functions on $K$. Let $M(K)=C(K)^{*}$ be the space of all complex-valued regular Borel measures on $K$ and let $M(K)_{1}^{+}$be the set of all probability measures in $M(K)$. Let $A \subset C(K)$ be a norm-closed subalgebra containing the constants and separating the points of $K$. Such algebras are called function algebras or uniform algebras (see Ga69] for basics of uniform algebras). If $m \in M(K)_{1}^{+}$, then the generalized Hardy space $H^{2}(m)$ associated to $A$ is the $L^{2}(m)$ closure of $A$. For any function $\phi \in L^{\infty}(m)$ the Toeplitz operator $T_{\phi}: B\left(H^{2}(m)\right) \rightarrow B\left(H^{2}(m)\right)$ is defined by $T_{\phi} h=P_{H^{2}(m)}(\phi h)$ for $h \in H^{2}(m)$ where $P_{H^{2}(m)}$ is the orthogonal projection of $L^{2}(m)$ onto $H^{2}(m)$. We shall also consider the usual multiplication operators $M_{\phi}$ defined on $L^{2}(m)$ by $M_{\phi} f=\phi f$ for all $f \in L^{2}(m)$. Let $H^{\infty}(m)$ denote the intersection $H^{2}(m) \cap$ $L^{\infty}(m)$ which is a weak*-closed subalgebra of $L^{\infty}(m)$. If $B \subset L^{\infty}(m)$ is any unital subalgebra, we shall denote by $\mathcal{T}(B)$ the $C^{*}$ - subalgebra of $B\left(H^{2}(m)\right)$ generated by all Toeplitz operators $T_{\phi}$ with $\phi \in B$. We also denote by $\mathcal{C}(B)$ the closed ideal in $\mathcal{T}(B)$ generated by all operators of the form $T_{\phi} T_{\psi}-T_{\psi} T_{\phi}$ for arbitrary $\phi, \psi \in B$ 
and by $\mathcal{S C}(B)$ the closed ideal in $\mathcal{T}(B)$ generated by all operators of the form $T_{\phi} T_{\psi}-T_{\phi \psi}$ with $\phi, \psi \in B$.

For our purposes we need to introduce the following definition. We shall say that a family of functions $F=\left\{\phi_{j}\right\}_{j \in J} \subset C(K)$ is a spherical multifunction if it is at most countable and if $\sum_{j \in J}\left|\phi_{j}(x)\right|^{2}=1$ for every $x \in K$. We are now able to state the main result of this section.

Theorem 4.1. Let $K$ be a compact Hausdorff space and let $A \subset C(K)$ be a unital norm-closed subalgebra. Suppose there exists a family $\left\{F_{\alpha}\right\}_{\alpha \in \Gamma}$ of spherical multifunctions in $C(K)$ where each $F_{\alpha}$ is of the form $F_{\alpha}=\left\{\phi_{j, \alpha}\right\}_{j \in J_{\alpha}}$ with each $\phi_{j, \alpha} \in A$ and such that for each pair of distinct points $x, y \in K$ there exist an index $\alpha \in \Gamma$ and an index $j \in J_{\alpha}$ such that $\phi_{j, \alpha}(x) \neq \phi_{j, \alpha}(y)$. Then for any regular Borel probability measure $m$ on $K$ the following assertions hold true:

(1) A bounded operator $X \in B\left(H^{2}(m)\right)$ is a Toeplitz operator with symbol in $L^{\infty}(m)$ if and only if it satisfies the following equations for all $\alpha \in \Gamma$ :

$$
\sum_{j \in J_{\alpha}} T_{\phi_{j, \alpha}}^{*} X T_{\phi_{j, \alpha}}=X
$$

(2) A bounded operator $X \in B\left(H^{2}(m)\right)$ is of the form $X=T_{\psi}$ for some $\psi \in$ $H^{\infty}(m)$ if and only if it commutes with $T_{\phi_{j, \alpha}}$ for all $\alpha \in \Gamma$ and all $j \in J_{\alpha}$.

(3) There exists a short exact sequence of $C^{*}$-algebras

$$
0 \rightarrow \mathcal{S C}\left(L^{\infty}(m)\right) \hookrightarrow \mathcal{T}\left(L^{\infty}(m)\right) \stackrel{\pi}{\longrightarrow} L^{\infty}(m) \rightarrow 0
$$

such that $\pi\left(T_{\phi}\right)=\phi$ for all $\phi \in L^{\infty}(m)$. In particular the spectral inclusion $\operatorname{essran}(\phi) \subset \sigma\left(T_{\phi}\right)$ holds true for all $\phi \in L^{\infty}(m)$.

(4) There exists a short exact sequence of $C^{*}$-algebras

$$
0 \rightarrow \mathcal{C}(C(K)) \hookrightarrow \mathcal{T}(C(K)) \stackrel{\pi}{\longrightarrow} C(\operatorname{supp}(m)) \rightarrow 0
$$

such that $\pi\left(T_{\phi}\right)=\phi$ on $\operatorname{supp}(m)$.

Conversely, if $A \subset C(K)$ is a function algebra such that the spectral inclusion essran $(\phi) \subset \sigma\left(T_{\phi}\right)$ holds true for every $m \in M(K)_{1}^{+}$and for every $\phi \in L^{\infty}(m)$, then A contains a separating family of spherical multifunctions as defined above.

Proof. Let us denote, for each $\alpha \in \Gamma$ and each $j \in J_{\alpha}$ by $T_{j, \alpha}$ the Toeplitz operator with symbol $\phi_{j, \alpha}$. Since each tuple $\left\{\phi_{j, \alpha}\right\}_{j \in J_{\alpha}}$ is a spherical multifunction it follows easily that in this case $\mathcal{S}_{\alpha}=\left\{T_{j, \alpha}\right\}_{\alpha \in \Gamma}$ is a spherical isometry and that $\mathcal{F}=\left\{\mathcal{S}_{\alpha}\right\}_{\alpha \in \Gamma}$ is a commuting family of spherical isometries in $B\left(H^{2}(m)\right)$. The separation property imposed on these spherical multifunctions implies via the StoneWeierstrass theorem that the $C^{*}$-algebra generated in $C(K)$ by the union of all families $F_{\alpha}$ with $\alpha \in \Gamma$ equals $C(K)$ itself. In turn this implies that the set $\tilde{\mathcal{F}}$ of all the corresponding multiplication operators $M_{\phi_{j, \alpha}}$ on $L^{2}(m)$ is the minimal normal extension of $\mathcal{F}$. Therefore, using Theorem 1.2 we infer that every operator $X \in B\left(H^{2}(m)\right)$ satisfying the equation in (1) is the compression of a bounded operator $Y$ in the commutant of all operators $M_{\phi_{j, \alpha}}$. Therefore, $Y$ commutes with all multiplication operators $M_{\phi}$ with $\phi \in C(K)$ which implies that $Y$ itself is a multiplication operator with some function $\psi \in L^{\infty}(m)$ which shows that $X$ is a Toeplitz operator i.e. $X=T_{\psi}$. Conversely, any Toeplitz operator obviously satisfies these equations because $H^{2}(m)$ is invariant for all operators $M_{\phi_{j, \alpha}}$. This completes the proof of (1). Now, the proofs of (2), (3) and (4) follow easily from the previous considerations combined with Theorem 1.2. 
In order to prove the last assertion of the theorem we shall use a result from Jan88 which says the following. If a function algebra $A \subset C(K)$ satisfies the Toeplitz spectral inclusion essran $(\phi) \subset \sigma\left(T_{\phi}\right)$ for every $m \in M(K)_{1}^{+}$and every $\phi \in L^{\infty}(m)$, then every continuous non-negative function on $K$ can be uniformly approximated on $K$ by finite sums of the form $\sum_{k=1}^{n}\left|g_{k}\right|^{2}$ with $g_{k} \in A$ for $1 \leq k \leq n$. Let $\Gamma$ be the set of all $f \in A$ with $|f|<1$ on $K$. It then follows from the above mentioned result from Jan88, that for each $f \in \Gamma$ there exists a sequence $\left\{g_{j, f}\right\}_{j \geq 1}$ in $A$ such that $1-|f|^{2}=\sum_{j \geq 1}\left|g_{j, f}\right|^{2}$ on $K$. If we denote $g_{0, f}=f$, then it follows that $F_{f}=\left\{g_{j, f}\right\}_{j \geq 0}$ is a spherical multifunction. Moreover, since $\Gamma$ is separating, $\left\{F_{f}\right\}_{f \in \Gamma}$ is separating as well. This concludes the proof of Theorem 4.1.

This theorem applies in particular when $K$ is the dual of a discrete partially ordered abelian group $G$ and $A \subset C(K)$ is the uniform algebra generated by the evaluations on positive elements of $G$. For this case, items (1), (2), (4) from Theorem 4.1 and the spectral inclusion from (3) have already been proved in [Mu87 for the normalized Haar measure on $K$. As a matter of fact, the validity of the spectral inclusion at (3) is equivalent to the existence of the short exact sequence in (3); see Sun87.

Another example of function algebras that fit into the framework of Theorem 4.1 is the following. Let $\Omega \subset \mathbb{C}^{n}$ be a bounded strictly pseudoconvex domain, and let $A(\Omega)$ be the Banach algebra of all continuous functions on $\bar{\Omega}$ which are holomorphic on $\Omega$. It then follows from an embedding theorem for such domains (see Theorem 3 in [Lo85) that there exist a natural number $N>1$ and functions $f_{1}, \ldots, f_{N}$ in $A(\Omega)$ such that the function $F: \partial \Omega \rightarrow \mathbb{C}^{N}$ defined by $F(z)=\left(f_{1}(z), \ldots, f_{N}(z)\right)$ is oneto-one and takes $\partial \Omega$ into the unit sphere in $\mathbb{C}^{N}$. This shows that $A(\Omega)$ satisfies the hypothesis of Theorem 4.1 when restricted to $\partial \Omega$. In particular this applies to any bounded domain with $C^{2}$ boundary in the complex plane. For the case of finitely connected domains in $\mathbb{C}$ with analytic boundary, exact sequences of the form (3) and (4) were constructed in Ab74. Toeplitz operators on strictly pseudoconvex domains have been extensively studied over the past decades; see [BG81, Up96 and the references therein.

\section{REFERENCES}

[Ab74] M.B. Abrahamse, Toeplitz operators in multiply connected regions, Amer. J. Math. 96 (1974), 261-297. MR0361891 (50:14333)

[Ar75] W. Arveson, Interpolation problems in nest algebras, J. Funct. Anal. 20 (1975), no. 3, 208-233. MR0383098 (52:3979)

[Ar98] W. Arveson, Subalgebras of $C^{*}$-algebras. III. Multivariable operator theory, Acta Math 181 (1998), no. 2, 159-228. MR 1668582 (2000e:47013)

[At90] A. Athavale, On the intertwining of joint isometries, J. Operator Theory 23 (1990), no. 2, 339-350. MR1066811 (91i:47029)

[At98] - GKS decomposition and spherical dilations, J. Funct. Anal. 154 (1998), 117-129. MR.1066811 (91i:47029)

[AL96] K.R.M. Attele, A.R. Lubin, Dilations and commutant lifting for jointly isometric operators - a geometric approach, J. Funct. Anal. 140 (1996), no. 2, 300-311. MR1409039 (97i:47011)

[BG81] L. Boutet de Monvel, V. Guillemin, The Spectral Theory of Toeplitz Operators, Princeton University Press, Princeton, NJ; University of Tokyo Press, Tokyo, Annals of Mathematics Studies, 99, 1981. MR620794 (85j:58141)

[BP05] D. Beltiţă, B. Prunaru, Amenability, completely bounded projections, dynamical systems and smooth orbits, Integral Equations Operator Theory 57 (2007), no. 1, 1-17.

[BC70] C.A. Berger and L.A. Coburn, One parameter semigroups of isometries, Bull. Amer. Math. Soc. 76 (1970), 1125-1129. MR0265984 (42:893) 
[BCL78] C.A. Berger, L.A. Coburn, A. Lebow, Representation and index theory for $C^{*}$-algebras generated by commuting isometries, J. Functional Analysis 27 (1978), no. 1, 51-99. MR0467392(57:7251)

[BH63] A. Brown, P.R. Halmos, Algebraic properties of Toeplitz operators, J. Reine Angew. Math. 213 (1963/1964), 89-102. MR0160136 (28:3350)

[CE77] M.D. Choi, E.G. Effros, Injectivity and operator spaces, J. Functional Analysis 24 (1977), no. 2, 156-209. MR0430809 (55:3814)

[Co73] L.A. Coburn, Singular integral operators and Toeplitz operators on odd spheres, Indiana Univ. Math. J. 23 (1973/74), 433-439. MR0322595 (48:957)

[DJ77] A.M. Davie, N.P. Jewell, Toeplitz operators in several complex variables, J. Functional Analysis 26 (1977), no. 4, 356-368. MR0461195 (57:1180)

[Did05] M. Didas, Spherical isometries are reflexive, Integral Equations Operator Theory 52 (2005), 599-604. MR2184607 (2006g:47009)

[Dix50] J. Dixmier, Les moyennes invariantes dans les semi-groups et leurs applications, Acta Sci. Math. Szeged 12 (1950), 213-227. MR0037470(12:267a)

[Do69] R.G. Douglas, On the operator equation $S^{*} X T=X$ and related topics, Acta. Sci. Math. (Szeged) 30 (1969), 19-32. MR.0250106 (40:3347)

[Do72] On the $C^{*}$-algebra of a one-parameter semigroup of isometries, Acta Math. 128 (1972), no. 3-4, 143-151. MR0250106 (40:3347)

[Do98] _ Banach Algebra Techniques in Operator Theory, Second edition. Graduate Texts in Mathematics, 179. Springer-Verlag, New York, 1998. MR0250106 (40:3347)

[Es99] J. Eschmeier, Algebras of subnormal operators on the unit ball, J. Operator Theory 42 (1999), 37-76. MR1694801 (2000c:47013)

[Es01] - On the structure of spherical contractions, Recent advances in operator theory and related topics (eds. L. Kerchy, C. Foias, I. Gohberg, M. Langer), pp. 211-242, Birkhauser, Basel (2001). MR1694801 (2000c:47013)

[Es06] - On the reflexivity of multivariable isometries, Proc. Amer. Math. Soc. 134 (2006), 1783-1789. MR1694801 (2000c:47013)

[EsP01] J. Eschmeier and M. Putinar, Some remarks on spherical isometries, "Systems, Approximation, Singular Integral Operators, and Related Topics" (A.A.Borichev and N.K.Nikolskii,eds.), Birkhauser, Basel et al., (2001), 271-292. MR.1882699 (2003e:47045)

[Ga69] T.W. Gamelin, Uniform Algebras, Prentice-Hall, Inc., Englewood Cliffs, N. J., 1969. MR0410387 (53:14137)

[Ito58] T. Itô, On the commutative family of subnormal operators, J. Fac. Sci. Hokkaido Univ. Ser. I 14 (1958), 1-15. MR0107177(21:5902)

[Jan88] J. Janas, Toeplitz spectral inclusion and generalized in modulus property, Proc. Amer. Math. Soc. 104 (1988), 231-234. MR958073 (90c:47045)

[Kad51] R. V. Kadison, Isometries of operator algebras, Ann. of Math. 54 (1951), 325-338. MR.0043392 (13:256a)

[Lo85] E. Løw, Embeddings and proper holomorphic maps of strictly pseudoconvex domains into polydiscs and balls, Math. Z. 190 (1985), 401-410. MR806898 (87b:32047)

[Mu87] G.J. Murphy, Ordered groups and Toeplitz algebras, J. Operator Theory 18 (1987), no. 2, 303-326. MR915512 (89f:46132)

[Mu92] Toeplitz operators on generalised $H^{2}$ spaces, Integral Equations Operator Theory 15 (1992), no. 5, 825-852. MR.915512 (89f:46132)

[St55] W.F. Stinespring, Positive functions on $C^{*}$-algebras, Proc. Amer. Math. Soc. 6 (1955), 211-216. MR0069403 (16:1033b)

[Sun87] C. Sundberg, Exact sequences for generalized Toeplitz operators, Proc. Amer. Math. Soc. 101 (1987), 634-637. MR911023 (89e:47040)

[TY78] J. Tomiyama and K. Yabuta, Toeplitz operators for uniform algebras, Tohoku Math. J. (2) 30 (1978)), no. 1, 117-129. MR0482220 (58:2302)

[Up96] H. Upmeier, Toeplitz Operators and Index Theory in Several Complex Variables, Operator Theory: Advances and Applications, 81. Birkhäuser Verlag, Basel, 1996. MR1384981 (97f:47022)

Institute of Mathematics "Simion Stollow" of the Romanian Academy, P.O. Box 1764, RO-014700 Bucharest, Romania

E-mail address: Bebe.Prunaru@imar.ro 